Supporting Information

\title{
Visualizing Oxidative Stress Level for Timely Assessment of Ischemic Stroke via a Ratiometric Near-Infrared-II Luminescent Nanoprobe
}

Meng Zhang $\dagger$, Zijun Wang $\dagger$, Caixia Wang $\ddagger$, Yuting Wut, Zhen $\mathrm{Li}^{*}, \ddagger$, and Zhihong Liu*, $\dagger$

+College of Chemistry and Molecular Sciences, Wuhan University, Wuhan 430072, China

‡College of Chemistry and Chemical Engineering, Hubei University, Wuhan 430062, China

*Corresponding author: Zhen Li; Zhihong Liu

Email: zhenli@hubu.edu.cn; zhhliu@whu.edu.cn 


\section{Table of Contents}

\section{Materials}

\section{Instrumentation}

Figure S1. Histograms of the size distribution of LnNPs.

Figure S2. UV-vis absorption spectra of IR-783.

Figure S3. Fluorescence emission spectra of IR-783 after reacting with ROS.

Figure S4-S8. Characterization of IR-LnNPs.

Figure S9. Fluorescence emission spectra of IR-783 loaded on the surface of LnNPs@VHPKQ.

Figure S10. Fluorescence emission spectra of IR-783 loaded on the surface of LnNPs@VHPKQ after reaction with HROS.

Figure S11. Selectivity of IR-LnNPs.

Figure S12. Dependence of the DCL intensity at $1550 \mathrm{~nm}$ of IR-LnNPs on the time of reaction with $\mathrm{HOCl}$ and $\bullet \mathrm{OH}$.

Figure S13. Thermodynamic stability of IR-LnNPs in different media.

Figure S14. Cell viability assay.

Figure S15-S16. Blood biochemical analysis and H\&E staining assays.

Figure S17. The assays of penetration depth for NIR-II luminescence imaging.

Figure S18. The upconverion luminescence spectrum of LnNPs.

Figure S19. Confocal imaging of bEnd.3 cells incubated with LnNPs@VHPKQ.

Figure S20. NIR-II luminescence imaging of the mice with IS.

Figure S21. MR imaging of the modeled mice.

Figure S22. Blood sugar level of the normoglycemia and hyperglycemia mice.

Figure S23. The change of LPO and SOD level in the serum. 
Materials. 1-Octadecene and oleic acid were purchased from Aladdin Reagent, Ltd. (Shanghai, China). Lipopolysaccharide (LPS) was obtained from Sigma-Aldrich. DSPE-PEG 2000 was acquired from Ponsure biological Co., Ltd. (Shanghai, China). DSPE-PEG 2000 -VHPKQHR was obtained from Ruixi biological technology Co., Ltd. (Xi-an, China). Other chemical reagents were purchased from Sinopharm Chemical Reagent Co., Ltd. (Shanghai, China). All of the reagents were analytical or better grade and used without further purification. All aqueous solution was prepared with ultrapure water (Mill-Q, Millipore, $18.2 \mathrm{M} \Omega \cdot \mathrm{cm}$ resistivity). Mice were supplied by SPF (Beijin) Biotechnology Co., Ltd. (Beijing, China). All animal studies were performed in accordance with Animal Care and Use Committee of Wuhan University.

Instrumentation. The size and morphology of LnNPs were characterized by the JEM2010 transmission electron microscope (TEM) operated at $200 \mathrm{kV}$. High-resolution TEM and corresponding elemental mapping of LnNPs were conducted on Talos F200S high resolution transmission electron microscopy (Thermo Fisher Scientific, USA). TEM images of LnNPs in the cells were operated at JEM 2100-plus transmission electron microscopy (JEOL, Japan). XRD measurement was acquired by an X-ray diffractometer (XRD, Bruker D8 Discover) with a $2 \theta$ range of $10^{\circ}-80^{\circ}$ with $\mathrm{Cu} \mathrm{K} \alpha$ irradiation $(\mathrm{k}=1.5406 \AA)$. FT-IR spectra were conducted on a Nicolet 5700 FTIR Spectrometer (Thermo Fisher Scientific, USA). UV-vis spectra were measured by UV2550 UV-vis spectrophotometer (Shimadzu, Japan). NIR-II fluorescence spectra were measured by an FLS1000 fluorometer (Edinburgh, UK). CCK8 test was conducted on an Mk3 microplate reader (Thermo Scientific Multiskan, USA). Confocal laser imaging was conducted on a Zeiss LSM 880 Microscope. In vivo NIR-II luminescence imaging was performed by an In-Vivo Master NIR-II fluorescence imaging system (Grand Imaging Technology Co. Ltd., Wuhan, China). 
A

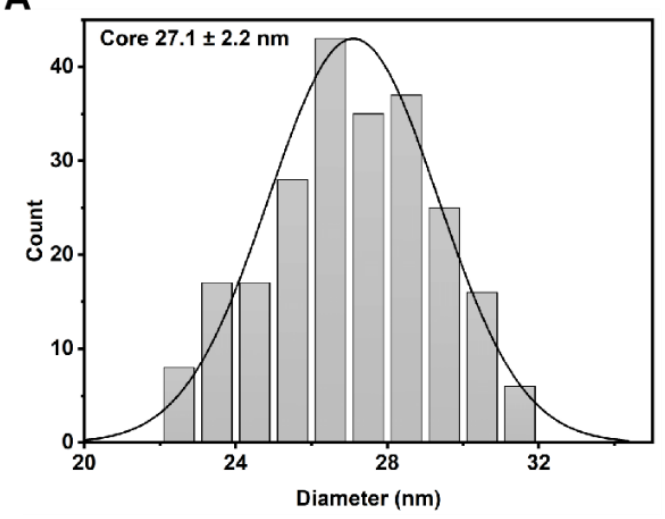

B

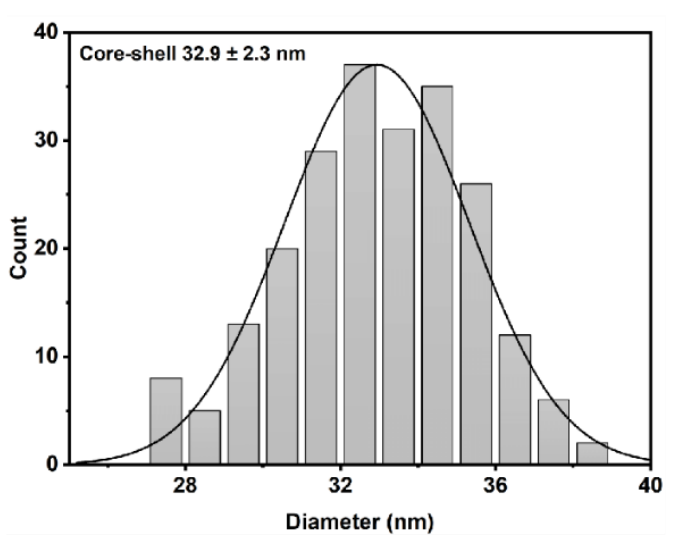

Figure S1. Histograms of the size distribution of core $\mathrm{NaYbF}_{4}: 5 \% \mathrm{Er}, 5 \% \mathrm{Ce}(\mathrm{A})$, and core-shell $\mathrm{NaYbF}_{4}: 5 \% \mathrm{Er}, 5 \% \mathrm{Ce} @ \mathrm{NaYF}_{4}: 20 \% \mathrm{Nd}$ nanoparticles (B).

A

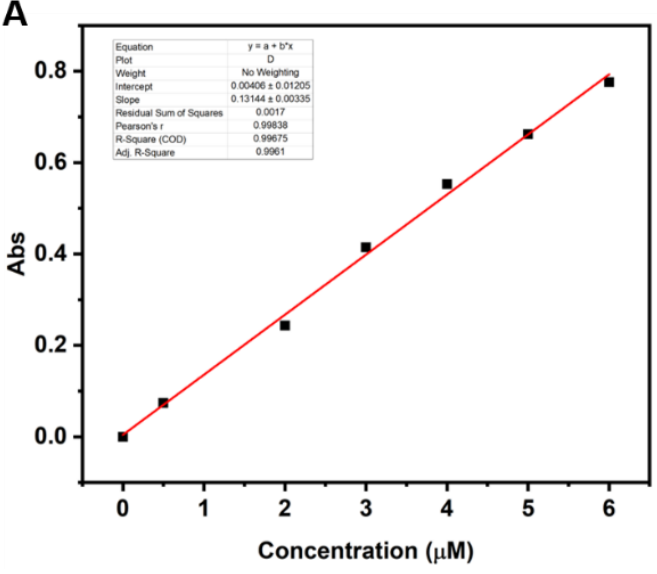

B

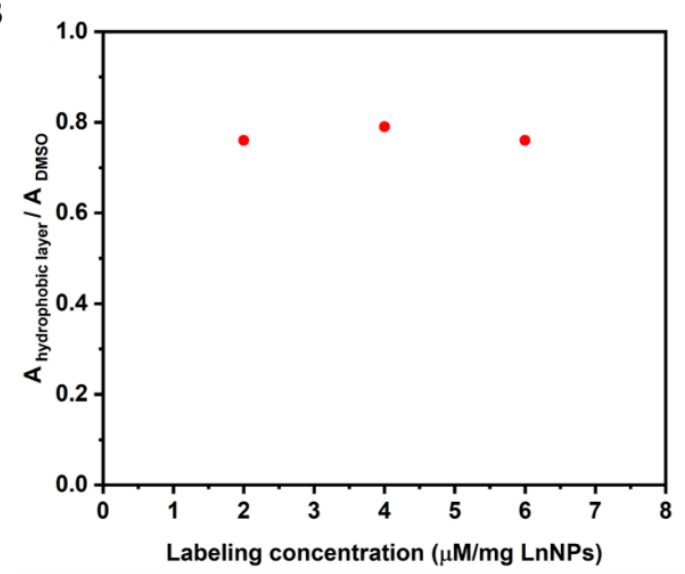

Figure S2. (A) Linear relationship between the absorbance at $808 \mathrm{~nm}$ and concentration of IR-783 in DMSO. (B) The ratio of the absorption of IR-783 loaded in the hydrophobic layer to that of the equal amount of IR-783 dissolved in DMSO. The average ratio is about 0.77 . 

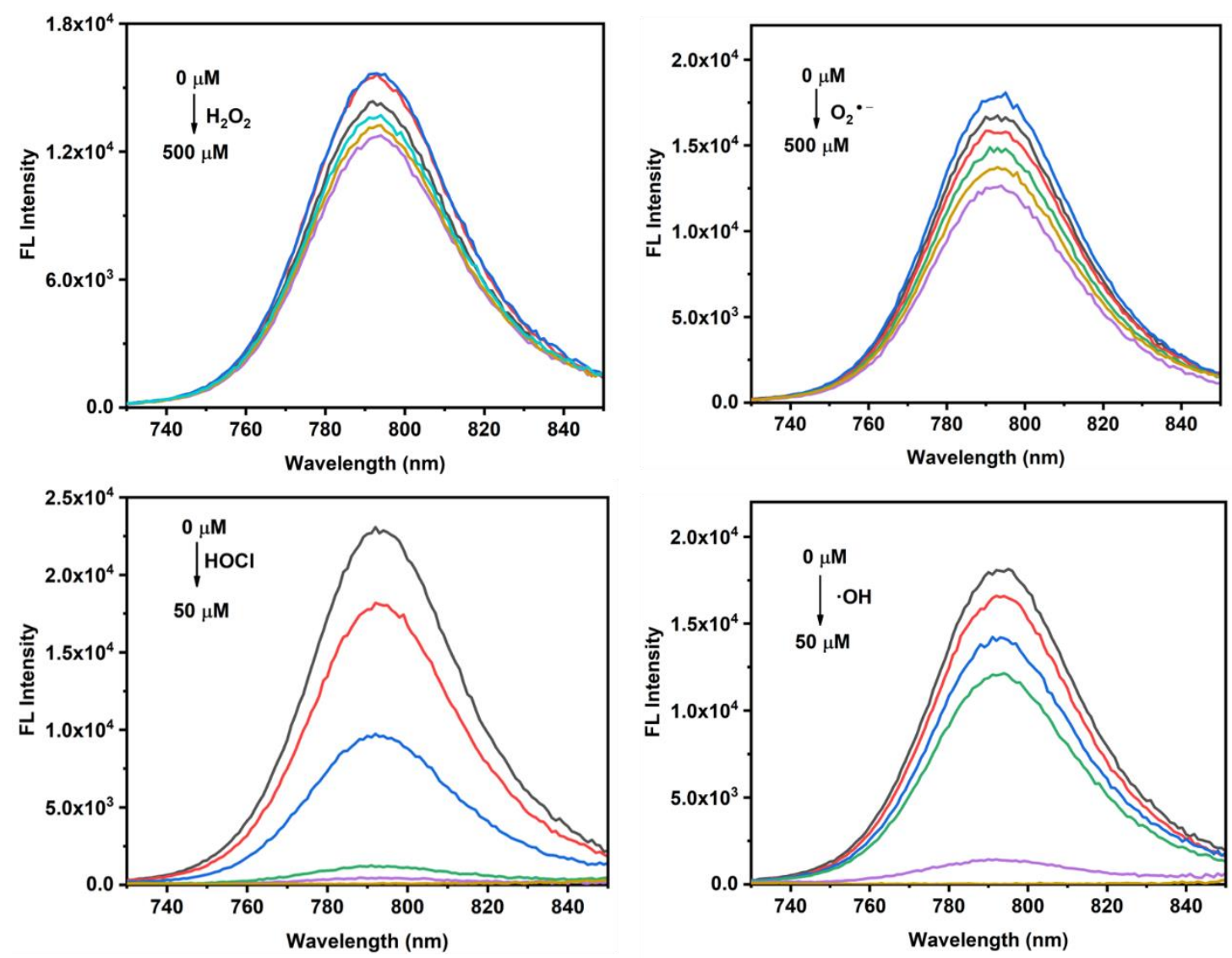

Figure S3. The fluorescence emission spectra of IR-783 after reacting with different concentrations of $\mathrm{H}_{2} \mathrm{O}_{2}, \mathrm{O}_{2}{ }^{--}$, $\mathrm{HOCl}$, and $\bullet \mathrm{OH}$.

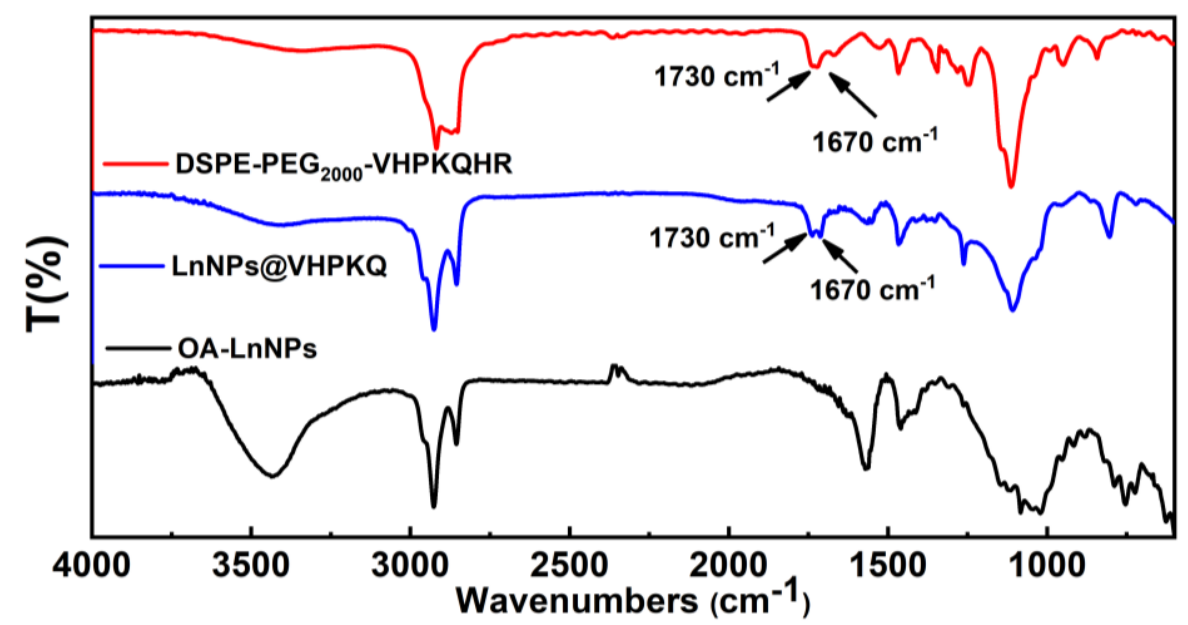

Figure S4. FTIR spectra of OA-LnNPs, LnNPs@VHPKQ, and DSPE-PEG $2000^{-}$ VHPKQHR. 


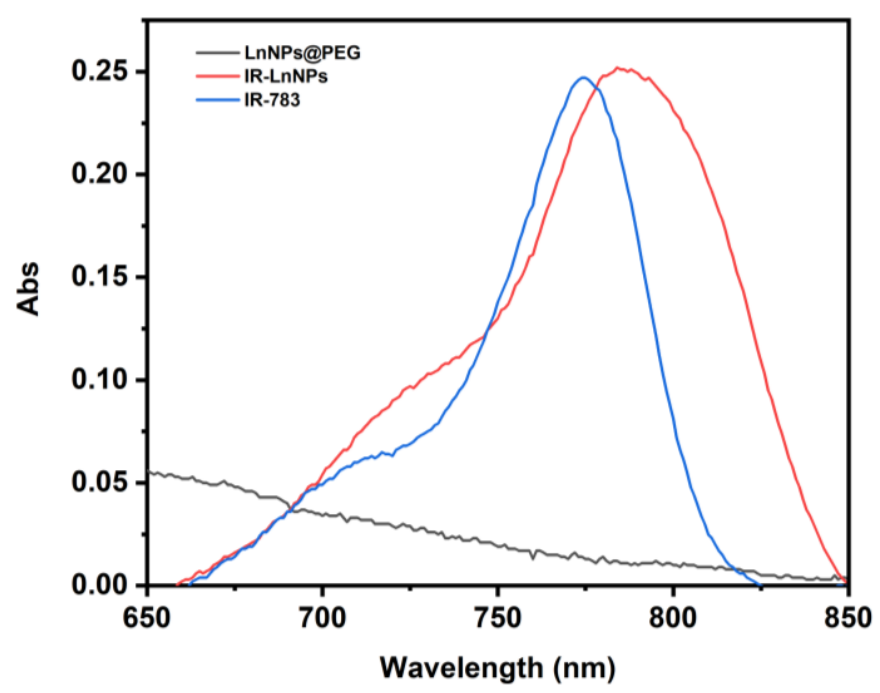

Figure S5. UV-vis absorption spectra of IR-783, LnNPs@PEG, and IR-LnNPs in the boric acid buffer ( $\mathrm{pH}=7.4,20 \mathrm{mM})$.

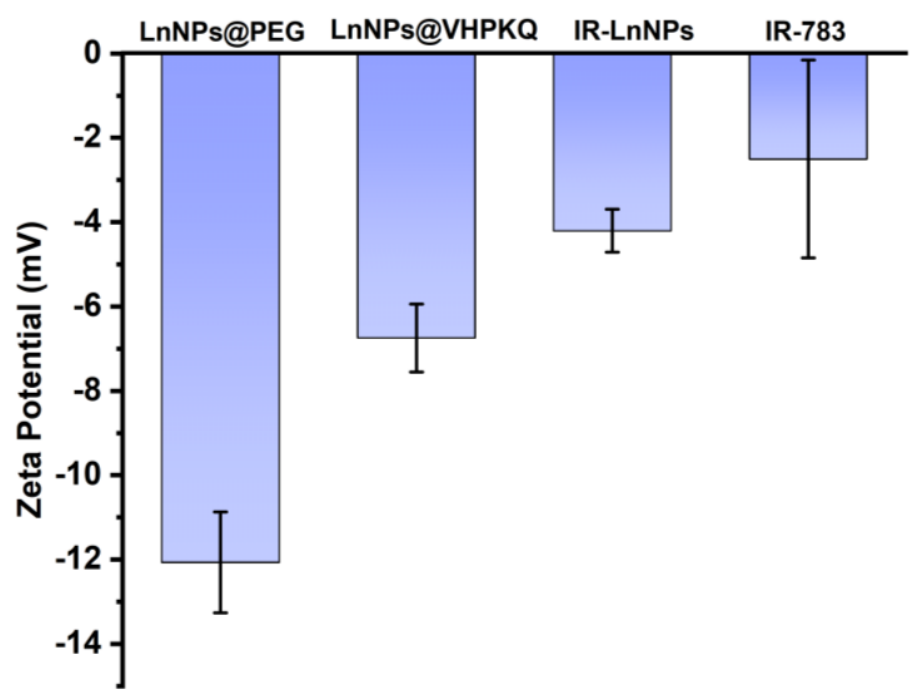

Figure S6. Zeta potentials of LnNPs@PEG, LnNPs@VHPKQ, IR-LnNPs and IR-783. 


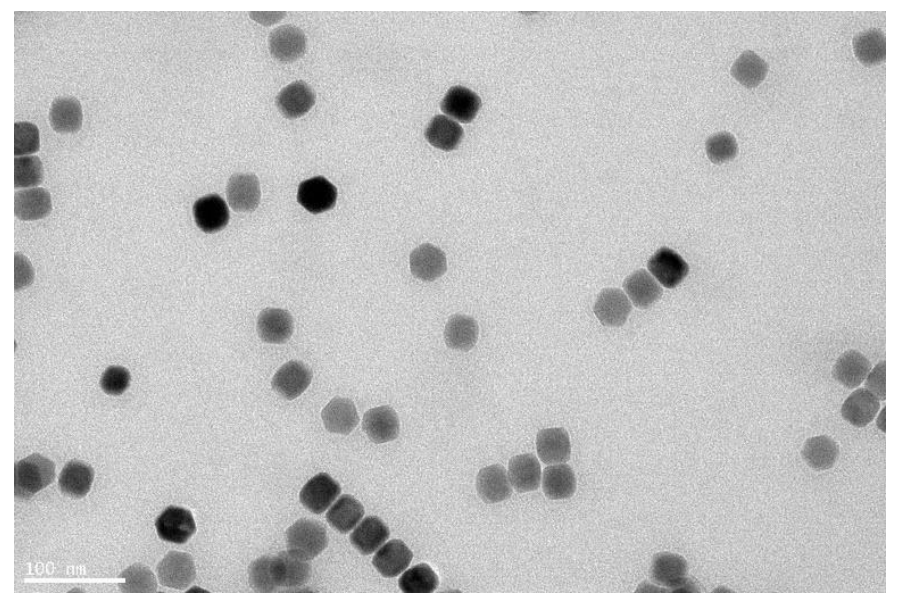

Figure S7. TEM image of IR-LnNPs.

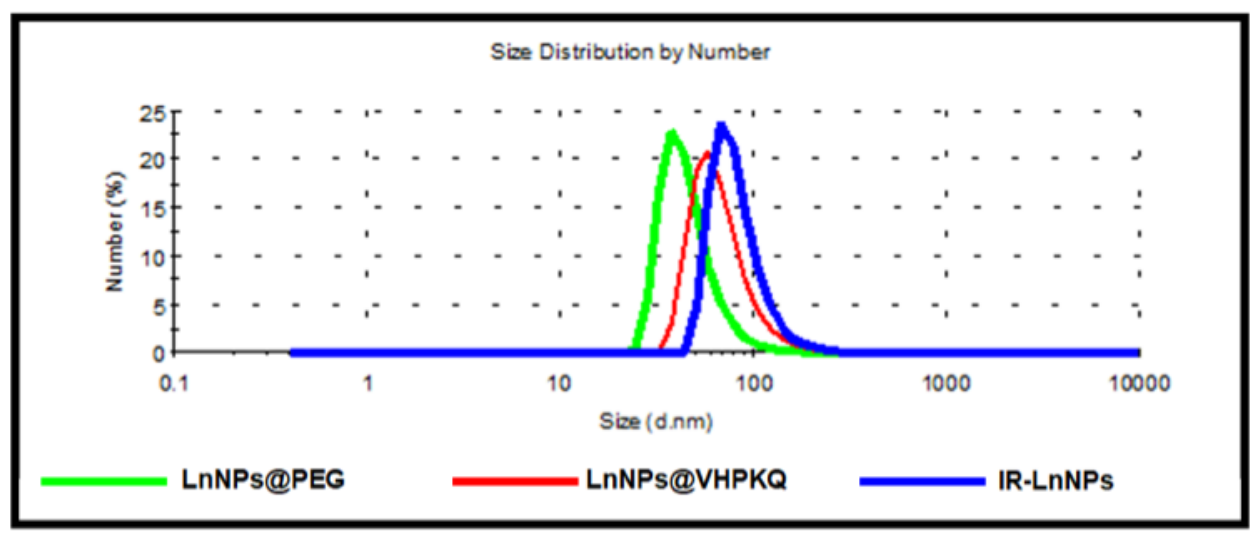

Figure S8. The change of the hydrodynamic diameter of LnNPs after a series of surface modification.

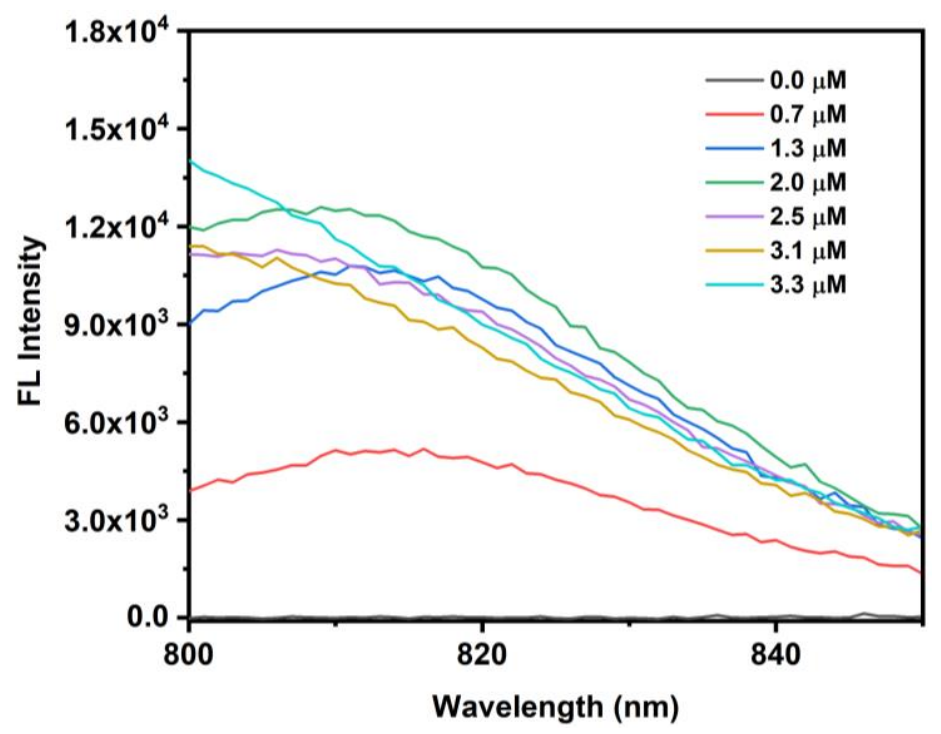

Figure S9. The fluorescence emission spectra of IR-783 with different concentrations 
loaded on the surface of LnNPs@VHPKQ.

A

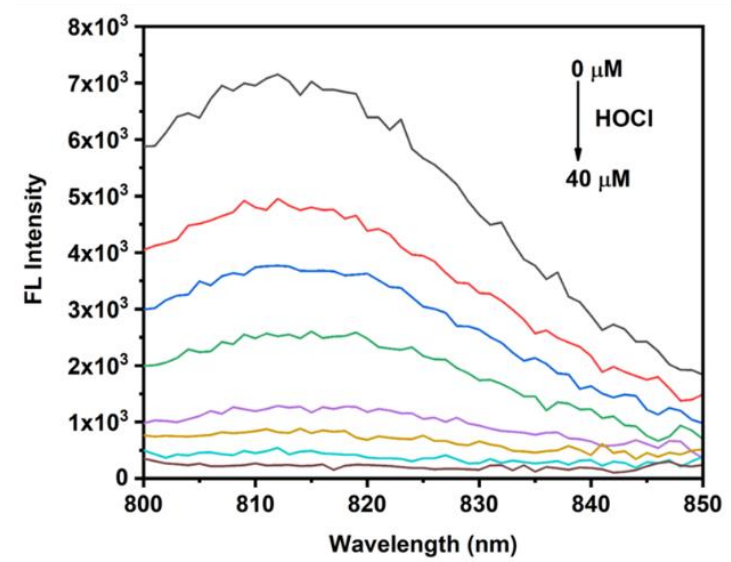

B

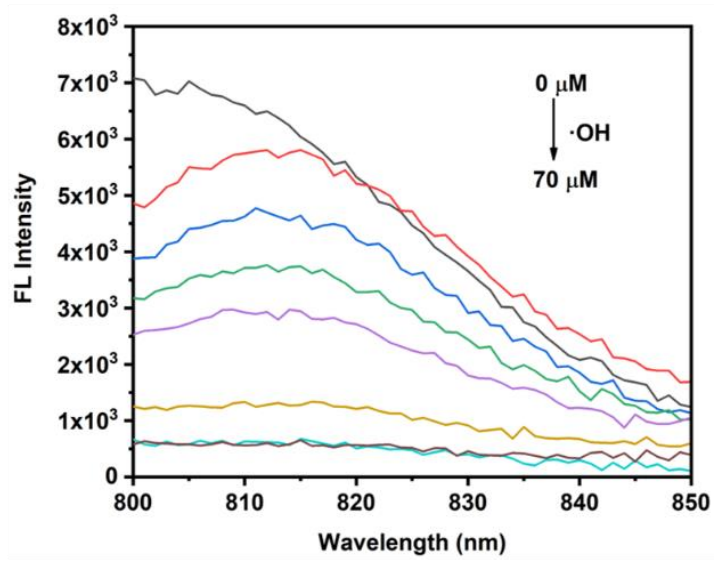

Figure S10. Fluorescence emission spectra of IR-783 loaded on the surface of LnNPs@VHPKQ after reacting with different concentrations of $\mathrm{HOCl}(\mathrm{A})$ and $\bullet \mathrm{OH}$ (B).

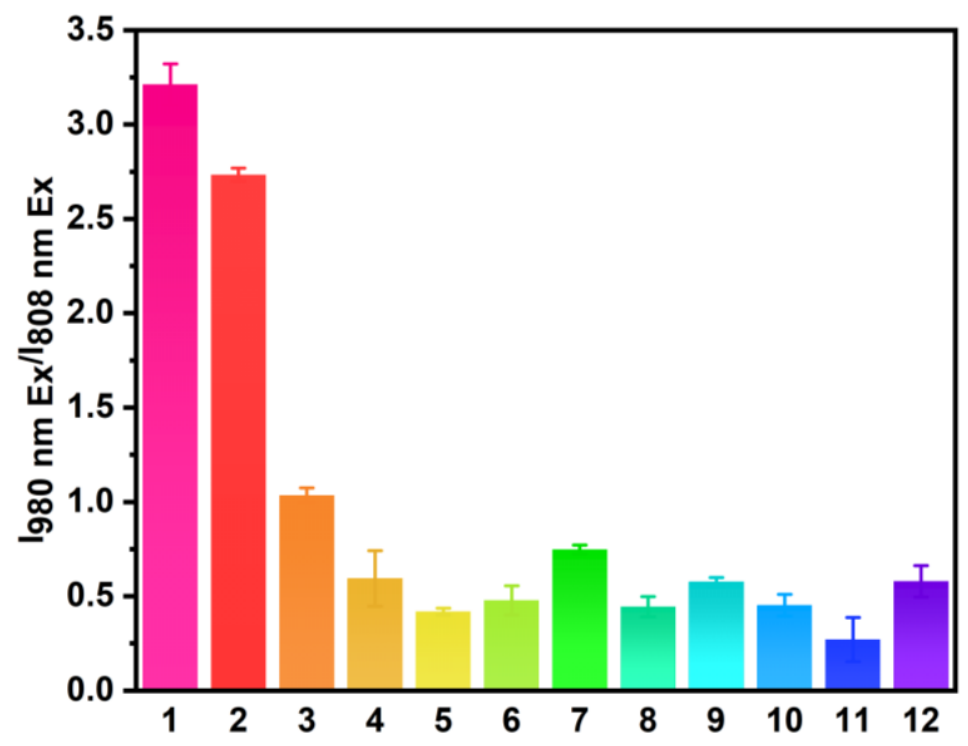

Figure S11. Ratiometric responses of IR-LnNPs $(0.4 \mathrm{mg} / \mathrm{mL})$ to $\mathrm{HOCl}, \cdot \mathrm{OH}$, and potential interferents (1: $40 \mu \mathrm{M} \mathrm{HOCl} ; 2: 60 \mu \mathrm{M} \bullet \mathrm{OH} ; 3: 50 \mu \mathrm{M} \mathrm{ONOO}^{-} ; 4: 500 \mu \mathrm{M}$ $\mathrm{H}_{2} \mathrm{O}_{2} ; 5: 500 \mu \mathrm{M} \mathrm{O}_{2}{ }^{--}$; 6: $500 \mu \mathrm{M} \mathrm{Cys;} \mathrm{7:} 500 \mu \mathrm{M} \mathrm{GSH}$; 8: $500 \mu \mathrm{M} \mathrm{Na} 2 \mathrm{~S} ; 9: 1 \mathrm{mM}$ BSA; 10: $10 \mathrm{mM} \mathrm{Ca}^{2+}$; 11: $10 \mathrm{mM} \mathrm{Zn}^{2+}$; 12: blank). 
A

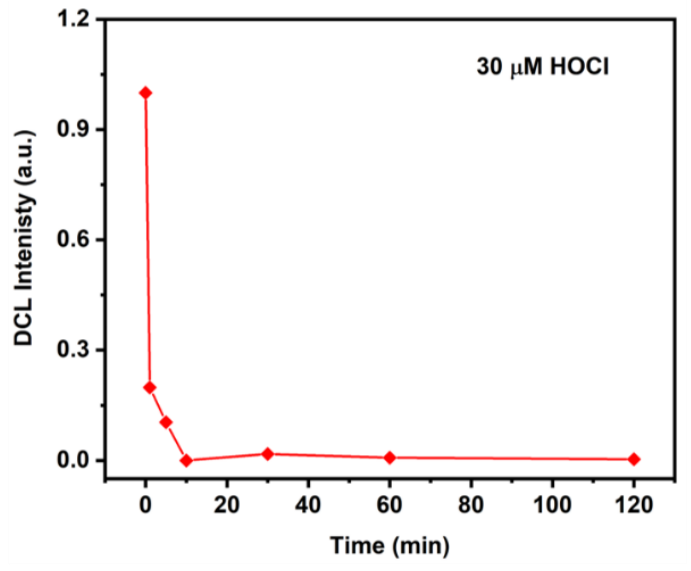

B

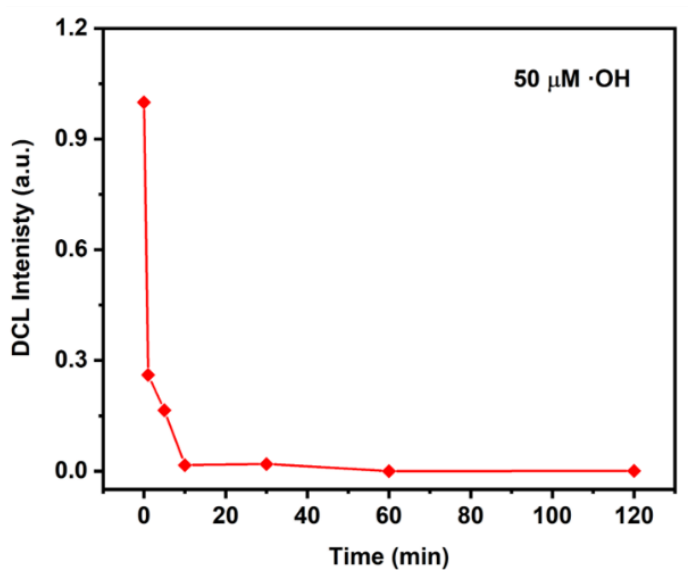

Figure S12. Dependence of the DCL intensity at $1550 \mathrm{~nm}$ of IR-LnNPs excited by 808 $\mathrm{nm}$ laser on the time of reaction with $30 \mu \mathrm{M} \mathrm{HOCl}(\mathrm{A})$ and $50 \mu \mathrm{M} \cdot \mathrm{OH}(\mathrm{B})$.

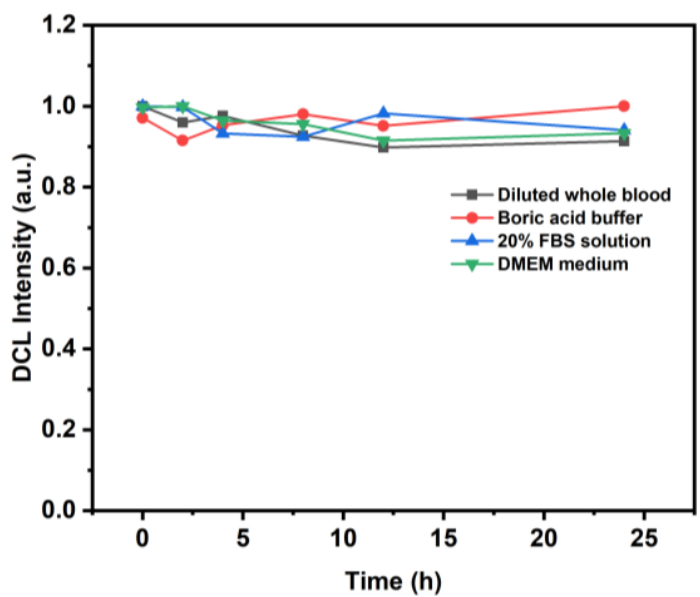

Figure S13. The DCL intensity of IR-LnNPs at $1550 \mathrm{~nm}$ under $808 \mathrm{~nm}$ excitation after incubation at $37^{\circ} \mathrm{C}$ in different media including 20 -fold diluted whole blood, boric acid buffer, 20\% FBS, and DMEM medium for different time points. 


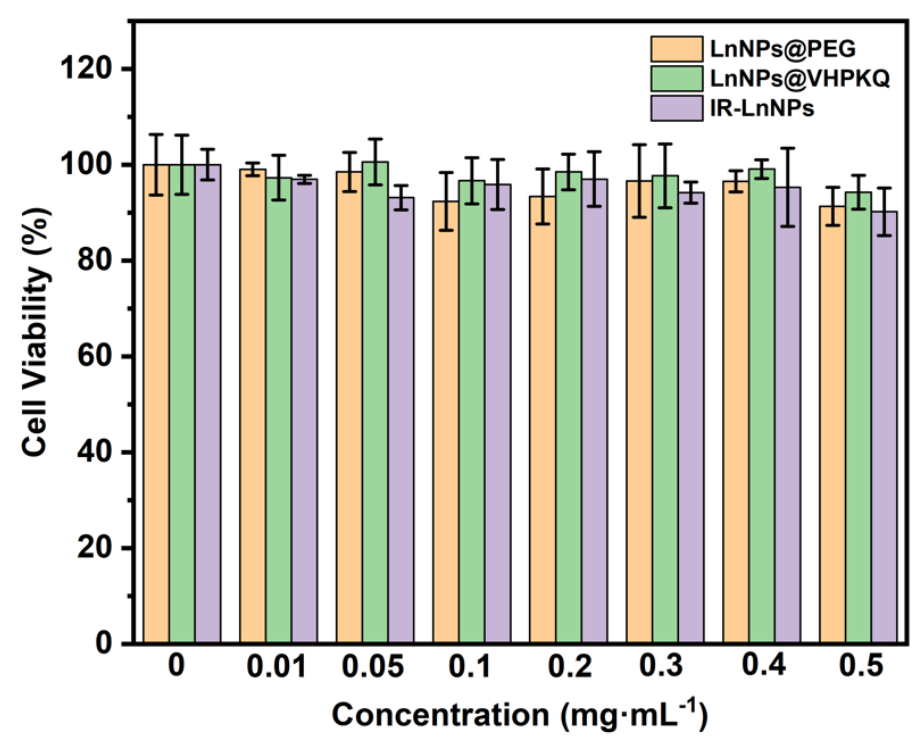

Figure S14. The viability of mouse brain microvascular endothelial cells (bEnd.3 cells) after incubation with different concentrations of LnNPs@PEG, LnNPs@VHPKQ, and IR-LnNPs for $24 \mathrm{~h}$.

A

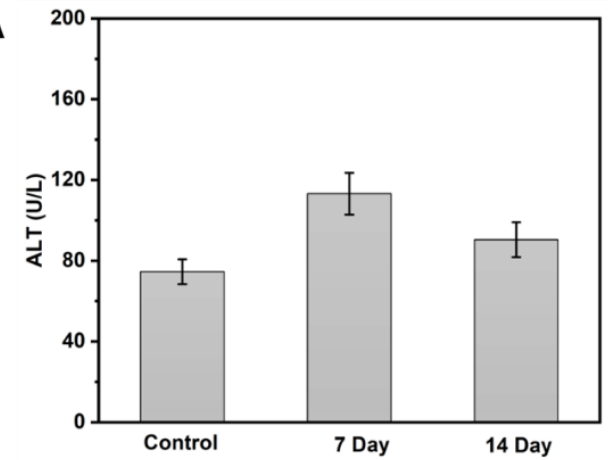

C

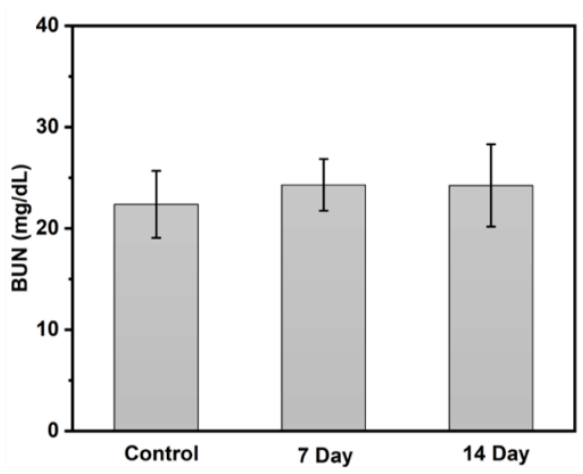

B
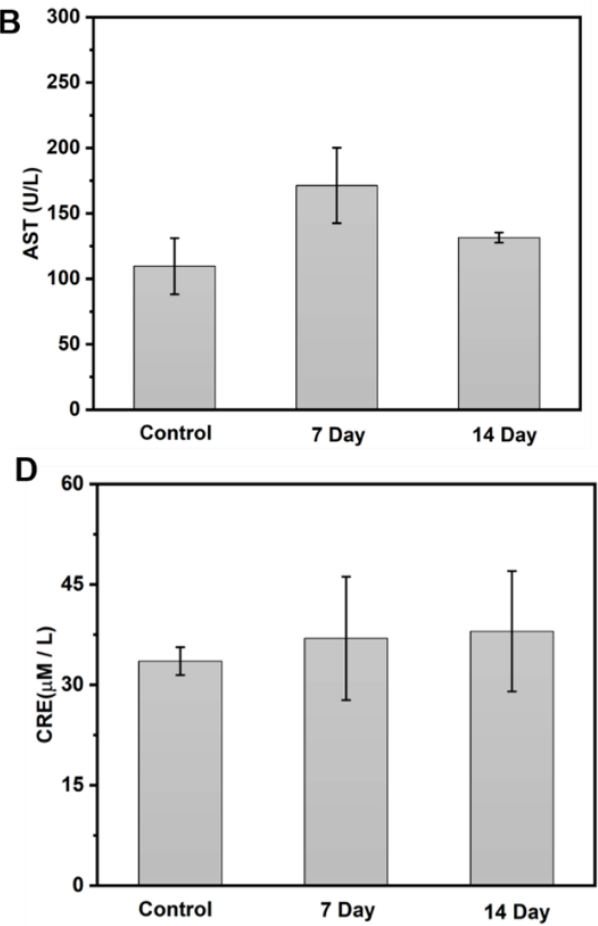

Figure S15. Blood biochemical analysis of the mice with or without IR-LnNPs treatment for 7 days and 14 days. (ALT: alanine aminotransferase; AST: aspartate aminotransferase; BUN: blood urea nitrogen; CRE: creatinine) 


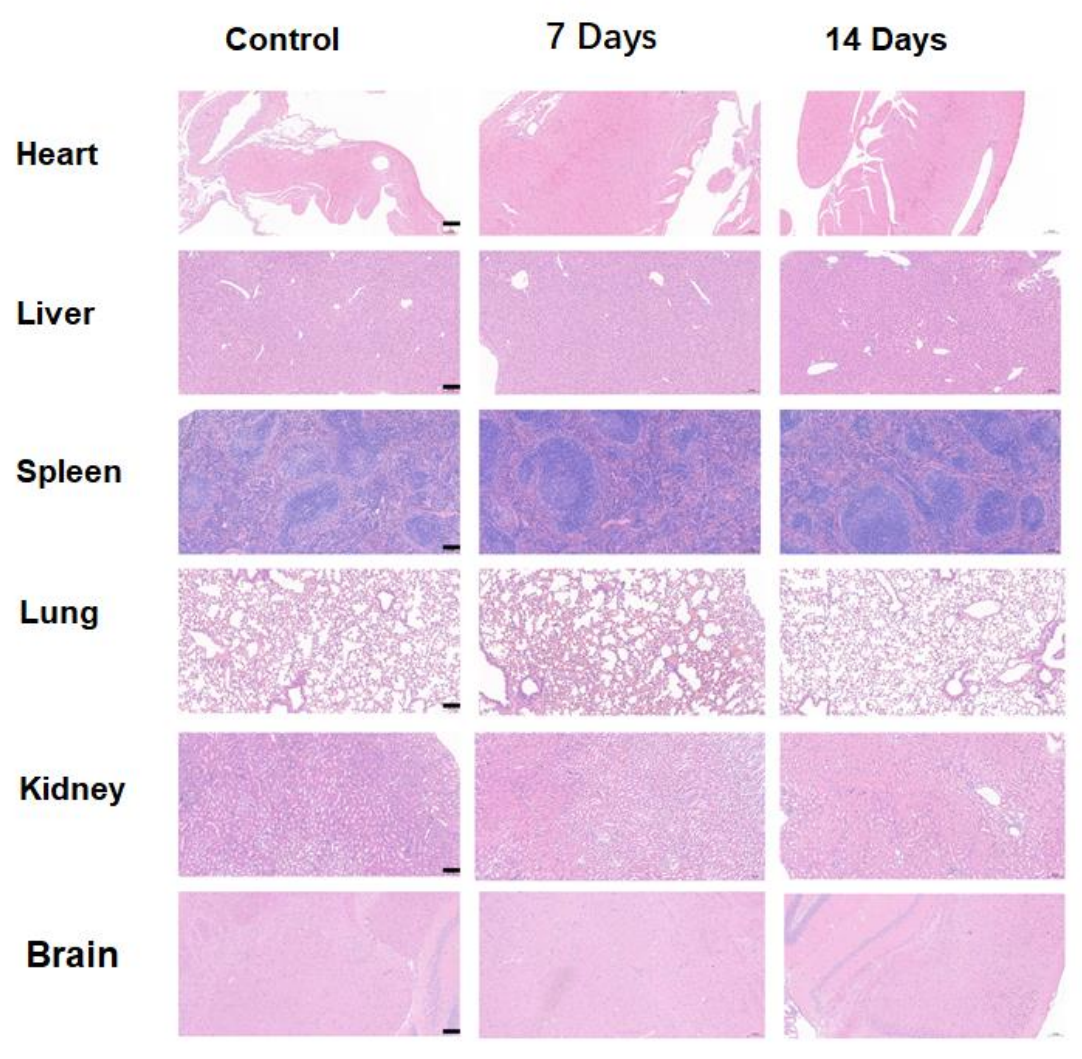

Figure S16. H\&E staining of major organs in mice with or without IR-LnNPs treatment for 7 days and 14 days. Scale bar: $100 \mu \mathrm{m}$.

A

\section{Penetration Depth (mm)}
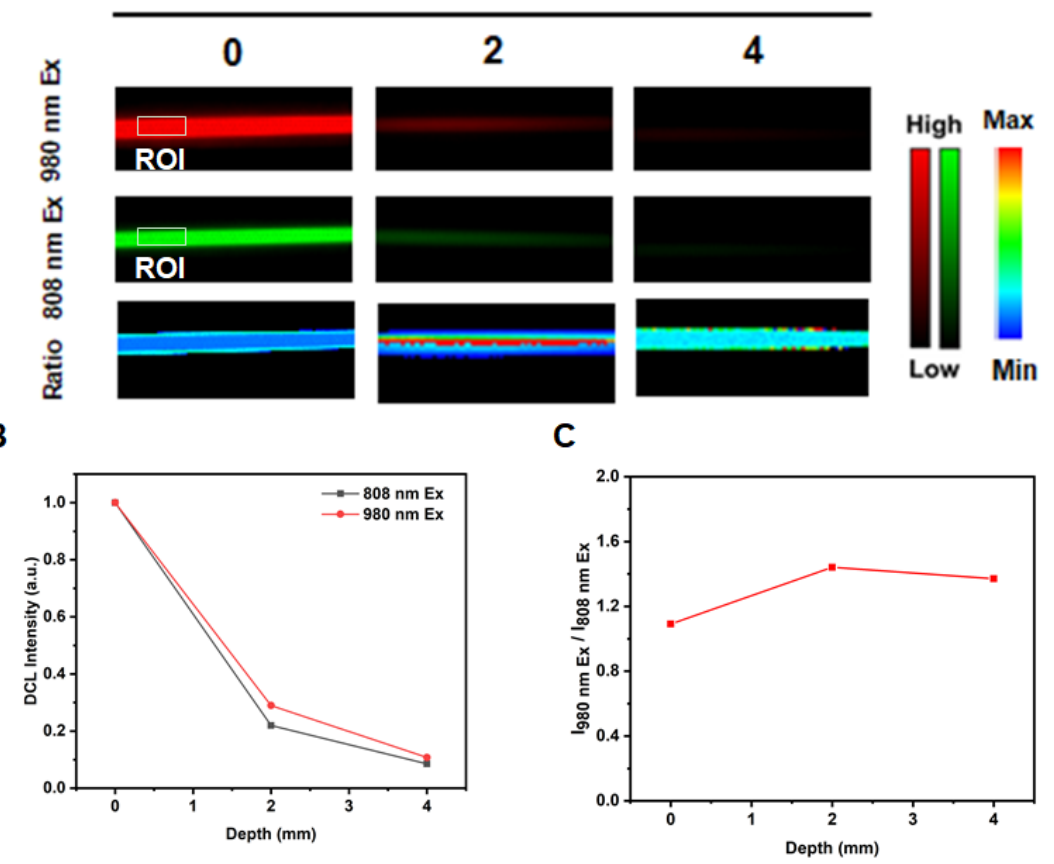

Figure S17. (A) NIR-II luminescence and ratiometric images of capillary covered with 
various height of $1 \%$ intralipid. (B) The normalized DCL intensities of capillary (marked in ROI region) under $980 \mathrm{~nm}$ and $808 \mathrm{~nm}$ excitation in (A). (C) The ratiometric values of $\mathrm{I}_{980 \mathrm{~nm} \mathrm{Ex}} / \mathrm{I}_{808} \mathrm{~nm}$ Ex at different penetration depth ( $\mathrm{I}_{980} \mathrm{~nm}$ Ex and $\mathrm{I}_{808} \mathrm{~nm}$ Ex represented as the mean luminescence intensity of the ROI regions, respectively).

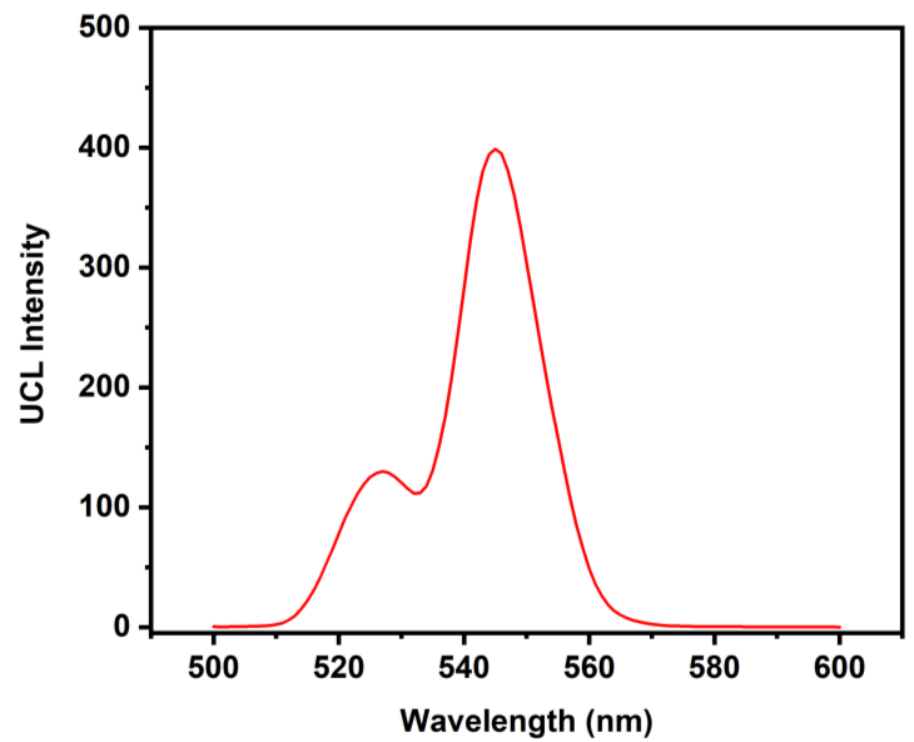

Figure S18. The upconversion luminescence (UCL) spectrum of LnNPs. 

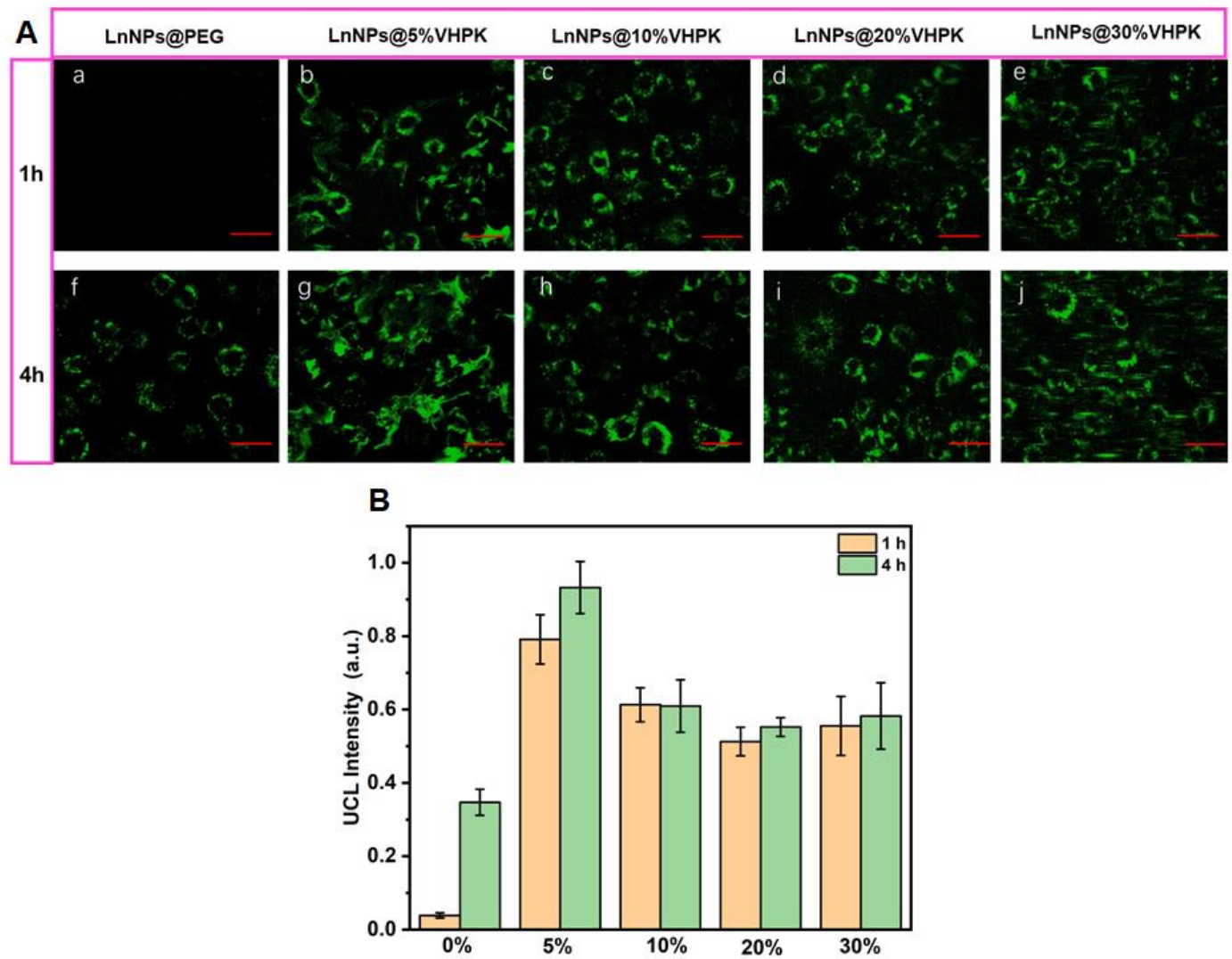

Figure S19. (A) Confocal images of bEnd.3 cells incubated with LnNPs@PEG, LnNPs@5\%VHPKQ, LnNPs@10\%VHPKQ, LnNPs@20\%VHPKQ, and LnNPs@30\%VHPKQ at $37{ }^{\circ} \mathrm{C}$ for $1 \mathrm{~h}$ and $4 \mathrm{~h}$, respectively. Images were acquired at 500-580 nm. Scale bar: $50 \mu \mathrm{m}$. (B) Normalized upconversion luminescence (UCL) intensities in (A).

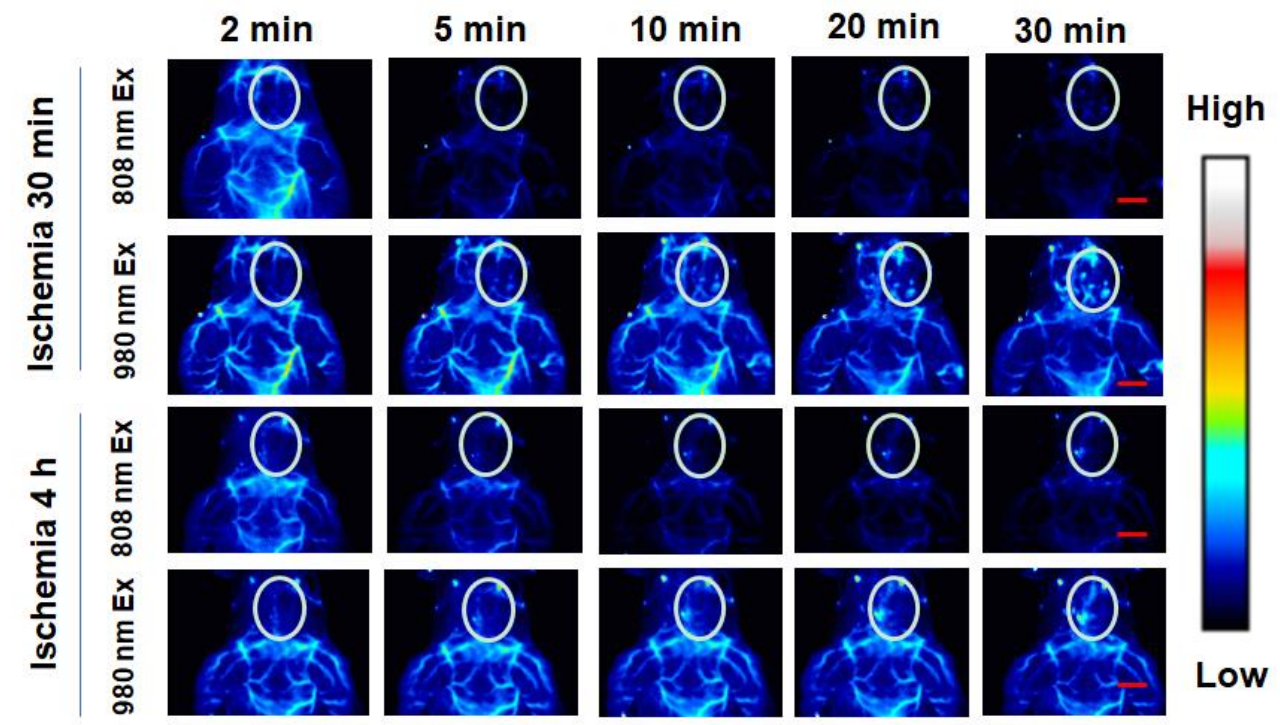


Figure S20. In vivo NIR-II luminescence imaging of the mice occurring cerebral ischemia for $30 \mathrm{~min}$ and $4 \mathrm{~h}$ after administration with the probes without decoration of VCAM-1 binding peptide (LnNPs@PEG-IR). Scale bar: 3 mm.
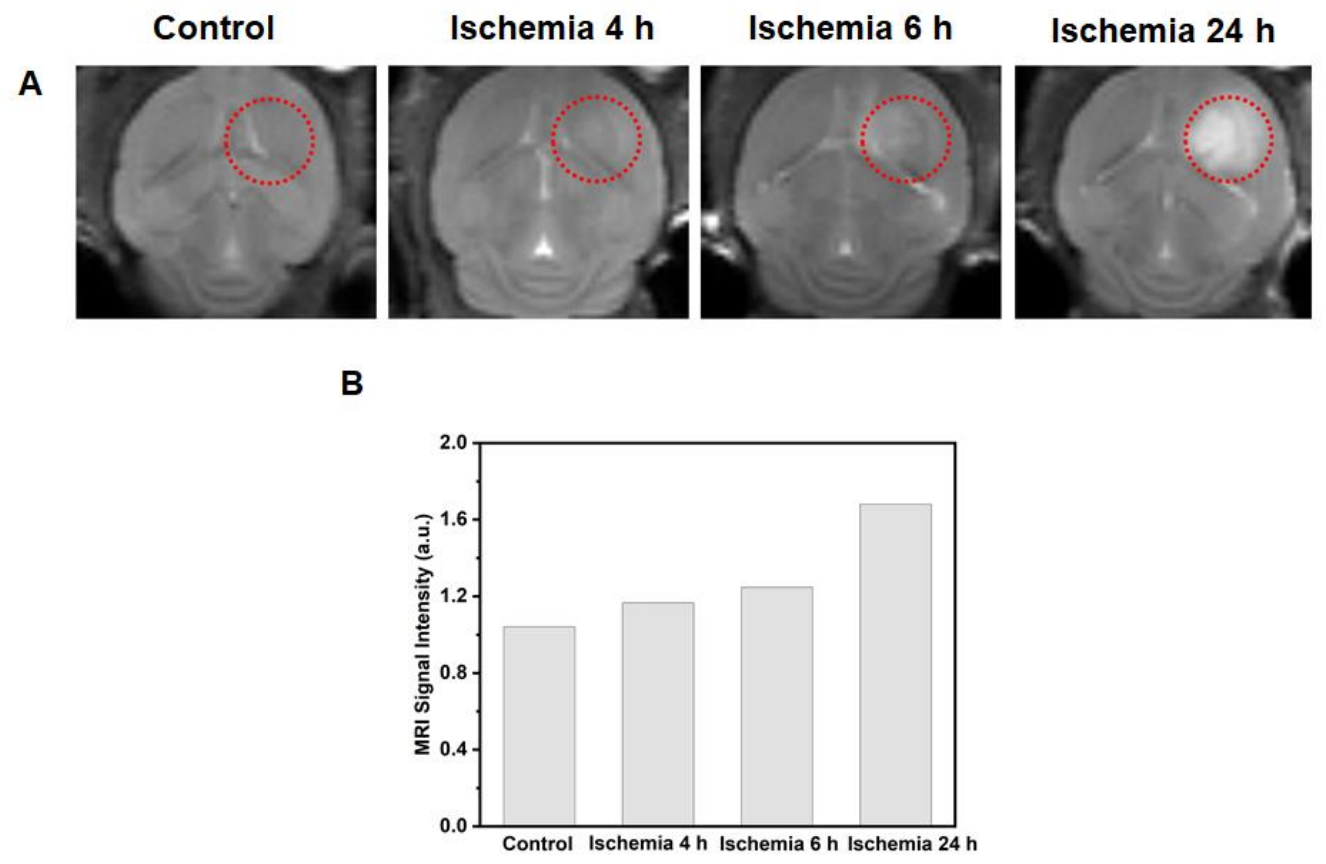

Figure S21. (A) Representative MR images of the IS mice at various time points. (B) The normalized MR signal intensities in (A).

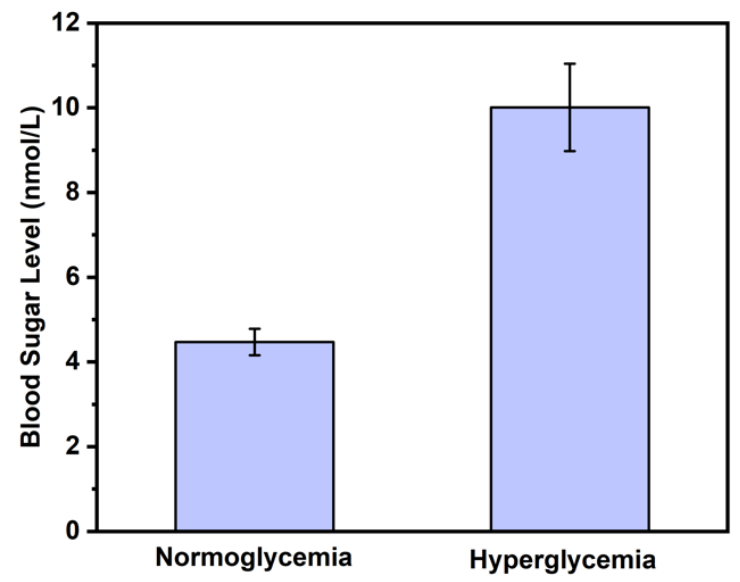

Figure S22. The blood sugar level of the normoglycemia and hyperglycemia mice. 

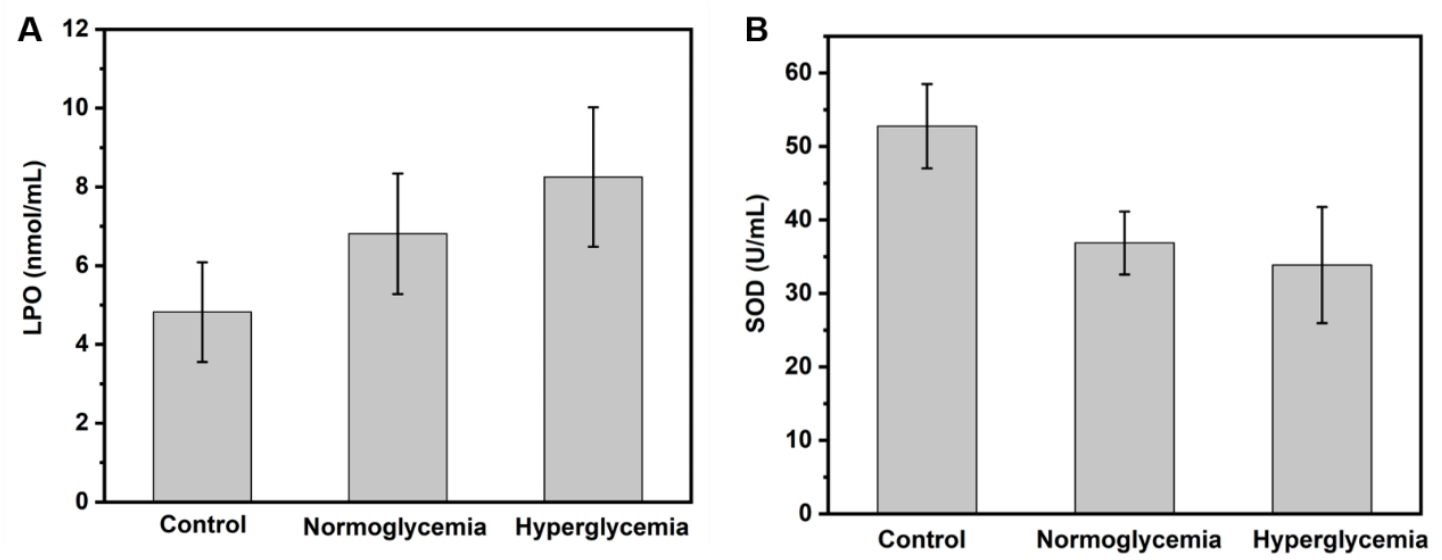

Figure S23. The change of superoxide dismutase (SOD) and lipid peroxidation (LPO) level of the normal mice, the normoglycemia mice with IS, and the hyperglycemia mice with IS. 